\title{
Characterization of DC current sensors under distorted conditions for railway applications
}

\author{
Helko E. van den Brom \\ Dept. Research \& Development \\ VSL, Dutch Metrology Institute \\ Delft, the Netherlands \\ hvdbrom@vsl.nl
}

\author{
Ronald van Leeuwen \\ Dept. Research \& Development \\ VSL, Dutch Metrology Institute \\ Delft, the Netherlands \\ rvanleeuwen@vsl.nl
}

\author{
Ralph Hornecker \\ Dept. Calibration \& Reference Materials \\ VSL, Dutch Metrology Institute \\ Delft, the Netherlands \\ rhornecker@vsl.nl
}

\begin{abstract}
To assess the reliability of the DC current measurement function on board trains and other electrical metering systems for transportation applications, a measurement setup has been realized to characterize the error of DC current sensors under realistic, distorted operating conditions. The device under test in this paper is a high-current shunt, but the setup is suitable for other types of current sensors as well. The highcurrent shunt under test was exposed to a variety of test signals, including current patterns as measured in a traction unit from Metro de Madrid during normal operation, to investigate the effect of dissipation on the error, the intrinsic current dependence, and the effect of AC distortion. From the results we conclude that the setup is a helpful and reliable facility to characterize DC current sensors for practical railway applications under realistic and harsh operating conditions.
\end{abstract}

Keywords-current sensor, current measurement function, energy measurement function, railway application

\section{INTRODUCTION}

In the last several years, public transport services have grown significantly, resulting in a growth in energy use and greenhouse gas emissions. Considering the overall annual energy consumption of railway systems and the EU target of reducing the related $\mathrm{CO}_{2}$ emissions by $50 \%$ by 2030 , a much more efficient use of energy is required. Energy reduction in railway systems can be realized by different means, including regenerative braking, energy-efficient driving, reduction of traction losses, optimization of comfort functions, energy metering, smart power management and renewable energy micro-generation [1]. In all cases, accurate and reliable knowledge of the energy consumption, regeneration or storage is essential to quantify the actual reduction obtained.

New European legislations require that, by 2019, energy billing is based on measurement equipment on board trains [2]. Accurately measuring the current on board trains, however, is challenging due to harsh conditions such as arching of the overhead lines, extreme temperatures, and rapid acceleration and deceleration of the train. These harsh conditions create all kinds of electrical distortion influencing the measurement of electrical parameters on board trains.

As set out in [3], the energy measurement function (EMF) that should be implemented on board trains provides the measurement of the consumed and regenerated energy of a traction unit with an accuracy on the order of $0.2 \%$ to $1 \%$. The
EMF consists of a voltage measurement function, a current measurement function and an energy calculation function. This paper focuses on the current measurement function, that measures the current taken from and returned to the contact line system. More specifically, the focus is on traction units designed for use on DC traction supply systems.

Usually in their datasheets, manufacturers of DC current sensors specify the operating range and accuracy only for static signals without distortion within a specific temperature range. In order to verify and test new or existing current measuring equipment under realistic operating conditions, a new measurement setup is developed to calibrate and characterize DC current sensors under adjustable non-ideal conditions. Such non-ideal operating conditions can be simulated for railway applications or other electrical systems used in transportation, such as for aircraft, ship propulsion or road vehicles.

In this paper the operating principle of the setup is explained, and its capabilities are demonstrated. In specific, measurement results are presented characterizing a DC high-current shunt by means of direct comparison to a high-precision reference sensor.

\section{MEASUREMENT SETUP}

\section{A. Principle of operation}

The measurement setup to characterize DC current sensors under realistic operation conditions has been developed based on a current ratio measurement technique [4]. The basic idea of the new setup is that the sensor under test is compared to a reference sensor that is calibrated using a primary reference setup and therefore traceable to international measurement standards. The harsh operating conditions are mimicked by applying various non-ideal test signals.

Typically, two types of current sensors exist for application in electrical systems for transportation. The first type of sensor converts current to voltage, such as for example a current shunt for DC systems, or a Rogowski coil for AC systems. For this type of sensor, the transresistance or transimpedance value, which is the ratio between the output voltage and the input current for DC or AC systems respectively, is the relevant parameter to be determined.

The second type of sensor converts the high input current to a much lower output current, such as for example a zero-flux current sensor [5] with current output for DC systems, or a 


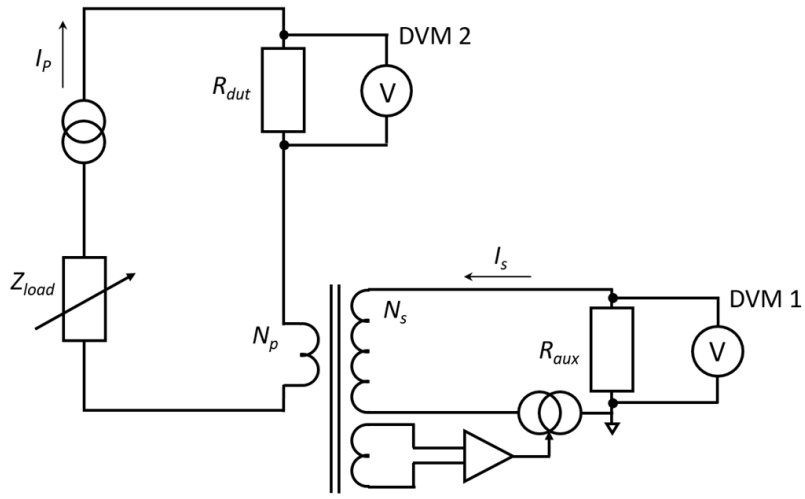

Fig. 1: Schematic of the measurement setup to characterize a DC current sensor. The device under test here is a high-current shunt $\left(R_{d u t}\right)$. The reference current sensor consists of a zero-flux current sensor in combination with an auxiliary high-precision shunt resistor $\left(R_{\text {aux }}\right)$.

current transformer for AC systems. For this type of sensor, the relevant parameter to be determined is the current ratio between input (or primary) current and output (or secondary) current. For these current-to-current transducers, an auxiliary high-precision current shunt can be used to convert the secondary current to voltage to be able to perform accurate measurements, or for direct comparison to current-to-voltage sensors. For simplicity, in this paper the combination of a current-to-current sensor and an auxiliary shunt will be considered a current-to-voltage sensor.

Fig. 1 shows a schematic of the measurement setup. The DC primary current $I_{p}$ is generated by means of a high-power DC current source (MagnaPower TSA5-900-HS ${ }^{1}$ ) and modulated using a programmable DC electronic load $Z_{\text {load }}$ (Chroma 63206A-150-600) that is connected in series. The current is applied to two current sensors in series. One of the sensors is the high-current shunt under test with resistance $R_{d u t}$ of nominally $100 \mu \Omega$ (Ohm-labs MCS), designed for measurements with currents up to $300 \mathrm{~A}$ and equipped with an active cooling fan. The other sensor is the reference sensor, consisting of a zero-flux current transformer (LEM ITN 900-S Ultrastab) in combination with an auxiliary high-precision current shunt $R_{\text {aux }}$ to convert the output current to voltage. The output voltage of the two sensors is measured using two high-precision digital voltmeters (DVM) (Agilent 3458A). Note that not only current-to-current sensors but also current-to-voltage sensors can be characterized using a second auxiliary high-precision shunt, or even complete current measuring systems including an ammeter or voltmeter, when leaving out the second DVM.

The present electronic load can be programmed to simulate various load profiles with unipolar currents up to $600 \mathrm{~A}$ with frequency components up to $20 \mathrm{kHz}$. Examples relevant for railway applications are DC current with AC distortion (ripple), DC current varying with time, or user-defined waveforms. In this

${ }^{1}$ Commercial equipment is identified in this paper only to adequately specify the experimental procedure. Such identification does not imply recommendation or endorsement way the device under test can be characterized under conditions that resemble normal operation conditions for the current sensor, found in traction units or in other electrical systems in transportation applications. Note that to mimic the difference between traction mode and regenerative mode the direction of the current flow must be reversed by interchanging the connections of the sensor under test.

\section{B. Sampling ratio measurements}

The ratio of the output voltages of the reference sensor and the device under test is measured using two high-precision digital voltmeters, either in DC volt mode, for DC voltages, or in sampling mode using an asynchronous sampling technique [6], when measuring AC distorted signals.

For AC distorted signals the DC component is defined as the zero-frequency component of the Fourier spectrum, which is equal to the average of all samples in the sampling time window. To avoid spectral leakage and thus low-frequency $\mathrm{AC}$ modulation of the DC component and measurement errors, the signal is resampled using spline interpolation at a rate that is an integer multiple of the repetition rate of the AC distortion.

When using the asynchronous sampling technique, the aperture time of the sampling voltmeters used is set to $100 \mu \mathrm{s}$ with a sampling rate of $8 \mathrm{kS} / \mathrm{s}$, taking 12000 samples in a single reading using a moving average of 10 readings. These numbers are used to ensure we cover a bandwidth up to $2.5 \mathrm{kHz}$, which is far more than sufficient for practical applications. (Note that there are no requirements on sampling frequency or ripple frequency defined in [3]). In DC volt mode, 3 measurements are performed per second.

\section{Reference current sensor}

As a reference sensor, a LEM ITN 900-S Ultrastab highprecision zero-flux current transformer is used with a nominal ratio of secondary to primary windings of 1500:1 and $900 \mathrm{~A}$ maximum primary current, in combination with a broadband 1 A current shunt to convert the output current to voltage. The DC current ratio of the zero-flux sensor and the intrinsic current dependence over a wide range of input currents were determined with an accuracy of better than 1 part in $10^{6}$ using a direct current comparator bridge that is originally designed for high-current low-resistance calibrations [7]. Since in this study the typical currents are much smaller than the 900 A maximum input, a two-turn primary winding is used to increase the sensitivity of the sensor. The broadband 1 A current shunt was designed to have very low AC-DC difference for frequencies up to $100 \mathrm{kHz}$ [8] and calibrated at DC using standard resistance calibration techniques with an accuracy better than 1 part in $10^{6}$.

The effect of AC ripple on the DC readout of the reference sensor was determined using a setup with an auxiliary sensor consisting of a similar combination of zero-flux current transformer and broadband current shunt [9]. The DC current was sent through both the reference sensor and the auxiliary

by VSL, nor does it imply that the equipment identified is necessarily the best available for the purpose. 
sensor, whereas the AC ripple signal was only sent through the reference sensor. This way we found the influence of $\mathrm{AC}$ distortion on the DC output reading of the reference sensor to be as low as a few parts in $10^{6}$.

\section{Definition of the measurand}

The current ratio error of a current-to-current sensor that converts a primary current $I_{p}$ to a secondary current $I_{s}$, is defined as

$$
\varepsilon=\frac{n \cdot I_{s}}{I_{p}}-1,
$$

where $n$ is the nominal current ratio of the current sensor. For a current-to-voltage sensor that converts an input current $I_{\text {in }}$ to an output voltage $V_{\text {out }}$, the transresistance error is defined as

$$
\varepsilon=\frac{V_{\text {out }}}{R_{\text {nom }} \cdot I_{\text {in }}}-1,
$$

where $R_{\text {nom }}$ is the nominal transresistance value of the sensor.

When comparing two sensors such as described in Fig. 1, the same input or primary current $I_{i n}$ or $I_{p}$ is sent through both sensors in series. Hence, from the definitions (1) and (2) one can derive that the error of the device under test (DUT), $\varepsilon_{d u t}$, can be expressed in terms of the ratio of the two measured secondary currents $I_{s}^{r e f}$ and $I_{S}^{d u t}$ or output voltages $V_{\text {out }}^{\text {ref }}$ and $V_{\text {out }}^{\text {dut }}$, the nominal current ratios of the reference sensor and the sensor under test $n_{\text {ref }}$ and $n_{d u t}$ or the nominal transresistance values $R_{\text {nom }}^{r e f}$ and $R_{\text {nom }}^{\text {dut }}$, and the error of the reference sensor, $\varepsilon_{r e f}$. For the situation described in Fig. 1, in which a current-to-voltage sensor is compared to a current-to-current sensor, the corresponding equation reads

$$
\varepsilon_{d u t}=1-n_{r e f} \cdot R_{\text {nom }}^{\text {dut }} \cdot \frac{I_{s}^{\text {ref }}}{V_{\text {out }}^{\text {dut }}}+\varepsilon_{\text {ref }},
$$

where the equality holds up to first order in the ratio errors. Hence, the transresistance error of the sensor under test can be read directly from the measured ratio of the secondary current and output voltage after a simple correction for the ratio error of the reference sensor. Note that (3) can be rewritten as

$$
\varepsilon_{d u t}=1-\frac{R_{\text {nom }}^{\text {dut }}}{R_{\text {nom }}^{\text {ref }}} \cdot \frac{V_{\text {out }}^{\text {ref }}}{V_{\text {out }}^{\text {dut }}}+\varepsilon_{\text {ref }},
$$

where now the combination of the current-to-current reference sensor and the auxiliary current shunt resistor $R_{\text {aux }}$ is considered a current-to-voltage sensor having a transresistance equal to $R_{\text {nom }}^{\text {ref }}=n_{\text {ref }} \cdot R_{\text {aux }}$.

\section{MEASUREMENT RESULTS}

The setup described in the previous chapter has been used to characterize a $100 \mu \Omega$ Ohm-labs MCS high-current shunt by comparison to a high-precision zero-flux reference sensor. First, a DC measurement is performed, showing the stability of the measurement results and the effect of heating by the applied current. Second, the current dependence has been determined, that is, the transresistance error is measured for different input currents. Third, as a special case of the latter, a pattern of current
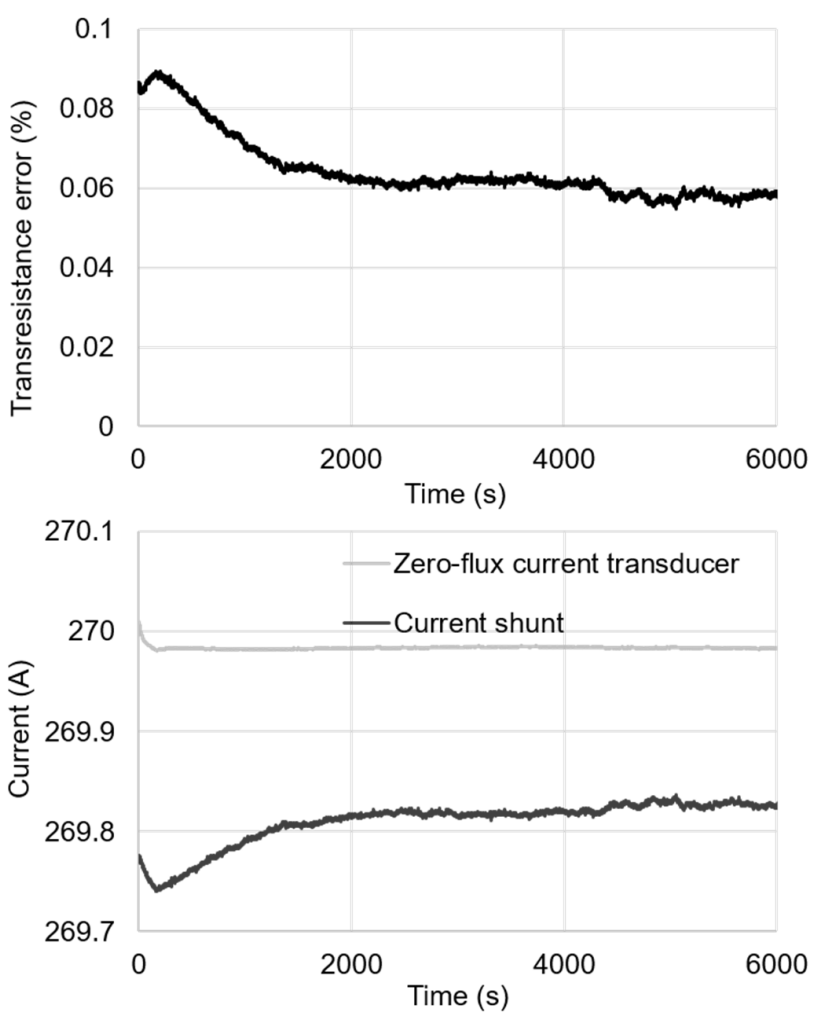

Fig. 2: The upper graph shows the measured transresistance error of the current shunt under test. The lower graph shows the corresponding current measured with the reference sensor (grey line) and the device under test (black line).

as a function of time which is typical for real situations in trains has been applied. Finally, the effect of AC ripple distortion on the DC output reading is determined.

The measurements mentioned in the previous paragraph are examples of measurements that can be carried out to demonstrate the possibilities and accuracy of the setup. Other specific measurements can be performed according to customer demands as well.

\section{A. DC calibration}

As a first measurement, the transresistance error of the current sensor under test is determined under a constant DC current of $270 \mathrm{~A}$, which is $90 \%$ of the maximum current. This measurement is performed to calibrate the transresistance value and to determine how continuous operation under high current affects the measurement results. The upper graph in Fig. 2 shows the error of the shunt as determined from the measured ratio between output signals of the reference sensor and the current shunt using (4). This graph shows that it takes considerable amount of time before the ratio reaches a stable value.

By analyzing the corresponding current measurements performed with the two sensors, as shown in the lower graph in Fig. 2, the drift observed in the ratio between both sensors is shown to be mainly caused by the current shunt. Both curves show a similar initial settling time of approximately 200 seconds, which can be attributed to the current source that needs 


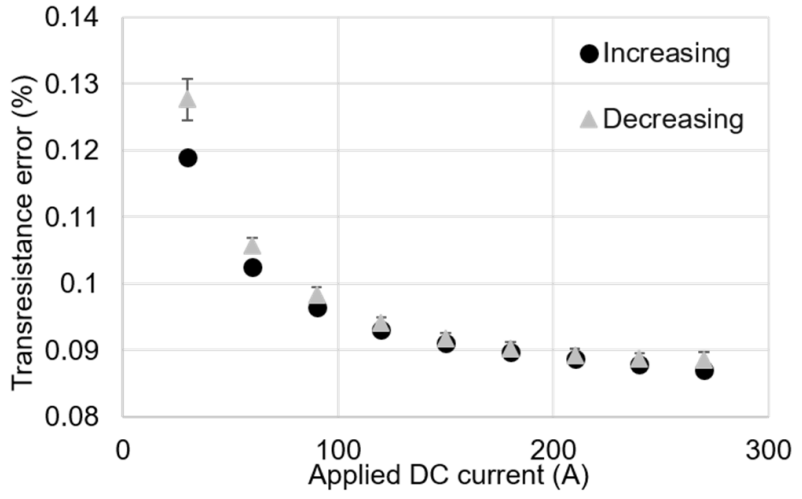

Fig. 3: Measured transresistance error of the current shunt under test as a function of the applied DC current. The solid circles and triangles represent measurements with increasing and decreasing current, respectively.

to recover from instantaneously being requested to deliver a large amount of current. After this initial settling of the current source, additionally, the current shunt requires about 2000 seconds to reach a stable value, which is about $0.03 \%$ different from its initial value. In the same time window, the reference sensor shows no drift at all. Hence, this slower drift observed in the error determination can be fully attributed to the high-current shunt under test and is most likely caused by dissipative selfheating, which is around $7.3 \mathrm{~W}$ for a 270 A current.

\section{B. Intrinsic current dependence}

The second measurement performed is to investigate possible intrinsic current dependence of the transresistance error, that is, current dependence caused by intrinsic properties of the shunt other than dissipation. In the previous section the effect of heating was discussed to take place on a time scale of a few hundred seconds. Therefore, to avoid heating, the intrinsic current dependence of the transresistance error is determined by measuring the output ratio of the two sensors while varying the level of the applied DC current at much shorter time scales. Since we know that the current dependence of the reference sensor is better than 1 part in $10^{6}$, any observed current dependence can be fully attributed to the sensor under test.

The electronic load is programmed with a current profile in which the current is alternately switched on for 5 seconds and switched off for 10 seconds. The current level is increased by 30 A with each step until a maximum of $270 \mathrm{~A}$ is reached. By applying the current for a short time and subsequently waiting, the effect of heating the sensor under test is expected to be limited. The whole sequence from $30 \mathrm{~A}$ to $270 \mathrm{~A}$ is repeated 5 times, with a few minutes in between. To test for possible hysteresis, the order of applied currents is reversed and a sequence with decreasing current is applied as well.

Fig. 3 shows the measured transresistance error of the device under test as a function of applied DC current. Each data point is the average of 5 independent measurements, taken in successive measurement sequences. The figure clearly shows that the sensor shows non-zero current dependence, in which the error increases with decreasing current. The largest transresistance error deviates by $0.03 \%$ from the smallest value
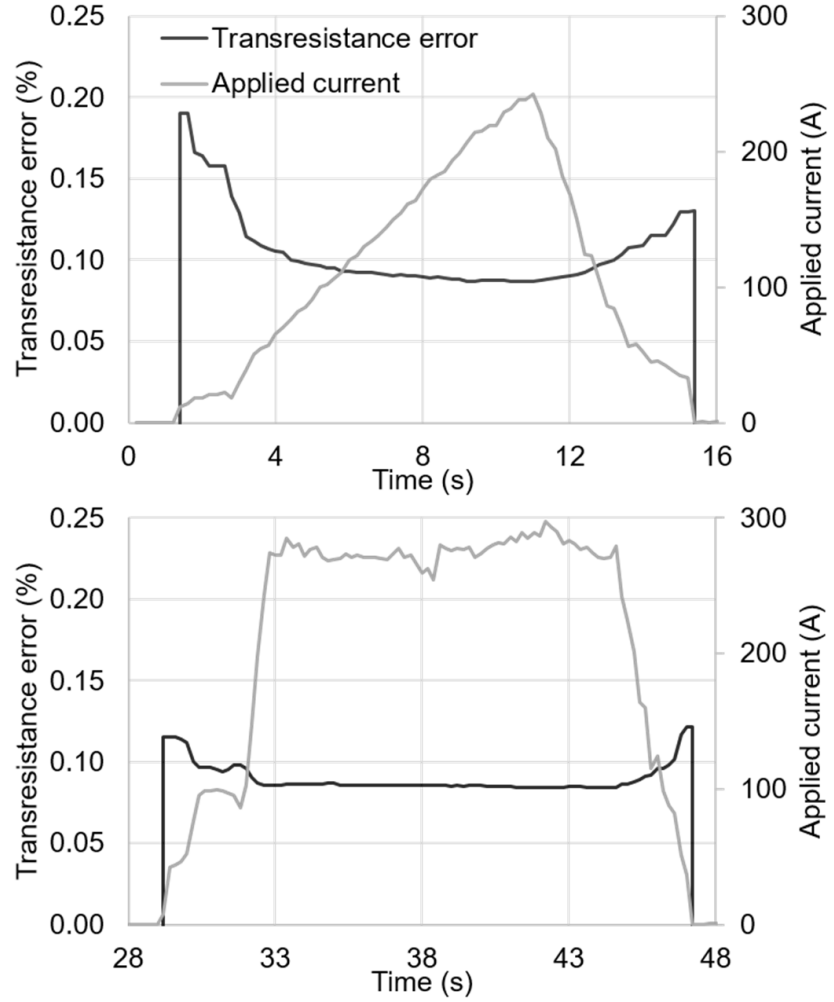

Fig. 4: Measured transresistance error of the current shunt under test (black line, left axis) during two simulated segments of a trip of an underground traction unit with the corresponding DC current applied (grey line, right axis).

measured at the largest current. The standard deviation of the measurements, shown as error bars in the figure, is typically a few parts per million only, which is hardly visible for many of the data points.

For decreasing current, somewhat different transresistance error values are measured, with larger standard deviations. This difference can still be attributed to a minor heating effect: when starting at high current, the shunt is heated somewhat more in the initial stage of the sequence already, and the corresponding (longer-term) drift already sets in. This results in a deviation especially at the lowest currents, because in the original increasing current measurement sequence the lowest current measurements hardly suffer from any heating at all. Additionally, in the decreasing current measurement sequence, the increased heating causes the last measurement at the smallest current to be performed at less well-defined internal temperature of the shunt, resulting in more spread in the data, as can be observed as larger error bars in the figure indeed.

\section{Realistic current patterns}

To mimic realistic operating conditions, a waveform is used that resembles the power consumption of a real traction unit. For this measurement we used voltage and current data recorded at the pantograph of a traction unit traveling between successive underground stations of Metro de Madrid. Also, the speed of the train was recorded simultaneously. The samples are recorded 
with a sample rate of $10 \mathrm{~S} / \mathrm{s}$. To apply the recorded data in our simulation, the current values have been scaled down by about six times in order not to exceed the current limit of $300 \mathrm{~A}$ of the current shunt under test. The corresponding patterns selected for loading the current sensors are programmed into the memory of the electronic load. Fig. 4 shows the corresponding applied current as a function of time as measured using the reference sensor as well as the error of the current shunt under test as deduced from the measured ratio between the output signals of the two sensors as defined in (4).

Two typical segments of a single trip between two successive train stations were simulated, having similar duration but showing different speed profiles and corresponding power consumption. During the first segment, taking about 13 seconds and represented in the upper graph in Fig. 4, the train accelerates with gradually increasing current, after which it comes into coasting mode while the current was gradually reduced to almost zero. The coasting mode itself is not investigated because it corresponds to almost constant current, which is less interesting for this investigation. During the second segment, taking about 17 seconds, recorded directly after the coasting mode and represented in the lower graph in Fig. 4, the applied current was quickly increased and subsequently remained for a considerable amount of time to its maximum value, corresponding to a further acceleration, after which the current was quickly reduced to zero.

The measurement results show that the transresistance error of the sensor under test depends on the amplitude of the applied current in the same way as observed in Fig. 3 and described in the previous section. In specific, the error is about $0.09 \%$ at a current of around $270 \mathrm{~A}$, whereas it is about $0.12 \%$ at a current around $100 \mathrm{~A}$. For smaller currents we even observe a further increase of the error. Furthermore, the first onset of the heating effect observed in section III.A might be observed already in the lower curve when maximum current is used continuously for more than 10 seconds.

\section{AC distortion}

To determine the effect of AC distortions on the measurement results, the electronic load is programmed with DC signals with and without additional AC component. In previous measurements it has been verified that DC measurements with the reference sensor are not influenced by $\mathrm{AC}$ distortions to within a few parts in $10^{6}$ [9].

Measurements are successively performed with and without applying the AC distortion signal. The effect of the AC distortion on the device under test $\delta_{d u t}^{a c}$ is then quantified by taking the difference between the error $\varepsilon_{d u t}^{d c+a c}$ as determined when applying DC with $\mathrm{AC}$ distortion and the error $\varepsilon_{d u t}^{d c}$ as determined when applying DC only,

$$
\delta_{d u t}^{a c}=\varepsilon_{d u t}^{d c+a c}-\varepsilon_{d u t}^{d c} .
$$

DC current of $200 \mathrm{~A}$ is applied with different AC distortion signals for the results presented in Fig. 5. The upper graph presents measurements for an AC signal with fixed peak-topeak magnitude of $25 \mathrm{~A}$ with frequency varying from $50 \mathrm{~Hz}$ to $2.5 \mathrm{kHz}$. From the results no frequency dependence can be
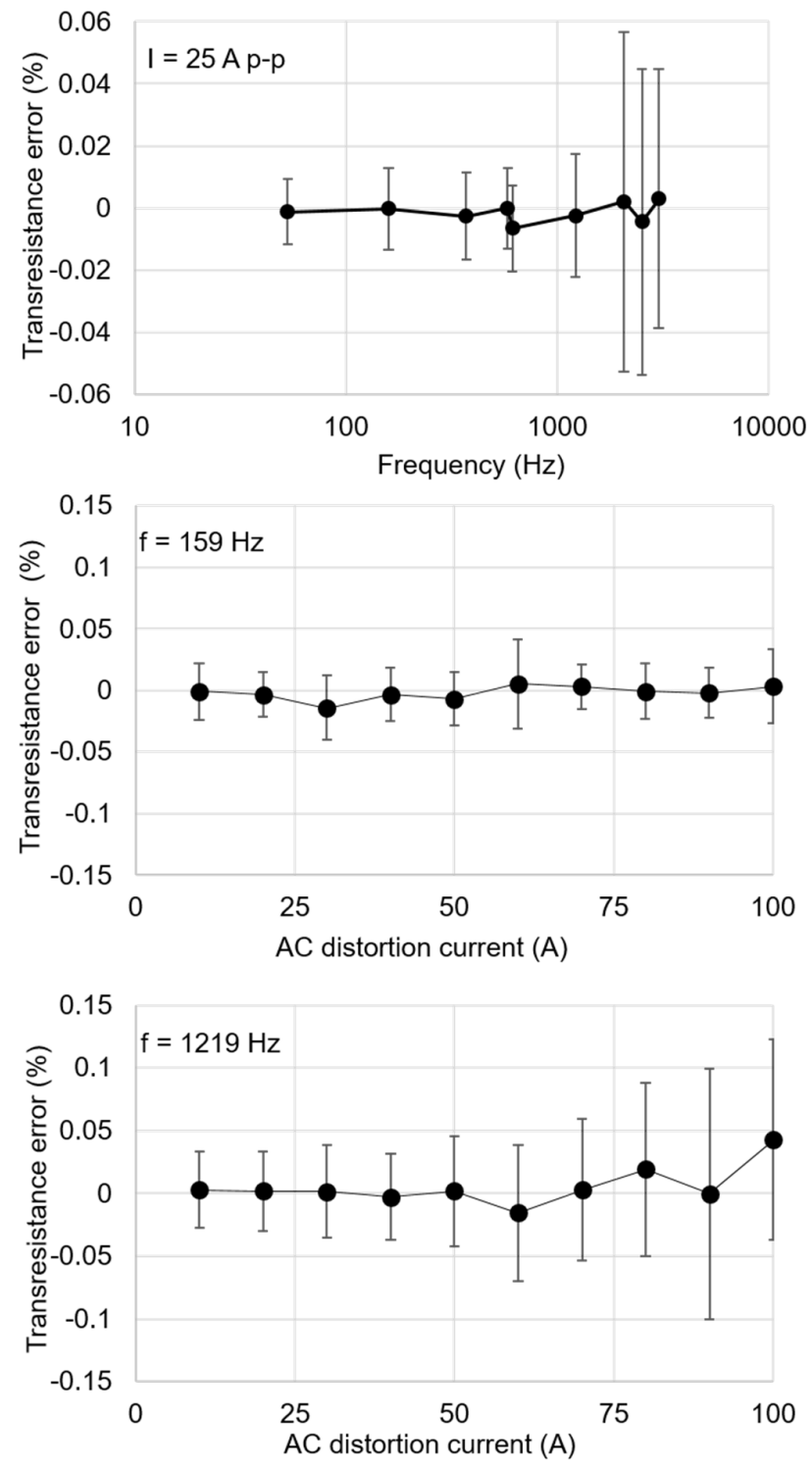

Fig. 5: Effect of AC distortion on the DC ratio measurement of the transresistance error of the current shunt under test as defined in (5). The upper graph shows the error as a function of frequency of the AC distortion for a fixed peak-to-peak magnitude of $25 \mathrm{~A}$. The lower two graphs show the error as a function of AC peak-to-peak magnitude for a fixed frequency of $159 \mathrm{~Hz}$ and $1219 \mathrm{~Hz}$, respectively.

observed to within the error bars representing the standard deviation of the measurements.

The middle and lower graphs in Fig. 5 represent measurements at fixed frequencies of $159 \mathrm{~Hz}$ and $1219 \mathrm{~Hz}$, respectively, in which the peak-to-peak magnitude of the AC signal is increased from $10 \mathrm{~A}$ to $100 \mathrm{~A}$ in steps of $10 \mathrm{~A}$. From the results no amplitude can be observed either, though the error bars are as large as $0.10 \%$ and one might argue that a nonnegligible effect is visible at the largest distortion current. On the other hand, this effect might be purely due to the earlier described heating effect. 


\section{SUMMARY AND CONCLUSION}

A measurement setup has been realized to characterize the error of the DC output of current sensors under realistic operating conditions for railway applications, i.e., with high distortion. The device under test in this paper is a high-current shunt, but the setup can deal with other types of current sensor or even complete current measurement systems as well. The sensor under test is compared to a reference sensor, consisting of a high-precision zero-flux current transformer in combination with a broadband high-precision shunt resistor. Distorted operating conditions are simulated using a programmable electronic load for currents up to $600 \mathrm{~A}$. For zero-flux current sensors which are commonly used in practice, this current can be increased by using multiple windings on the primary side.

The high-current sensor under test was exposed to a variety of test signals to investigate the effect of dissipation on the error, the intrinsic dependence on input current, and the effect of AC distortion. For the specific sensor under test in this paper, a $100 \mu \Omega$ high-current shunt designed for measurements with currents up to $300 \mathrm{~A}$ and equipped with an active cooling fan, the longer-term effect of dissipative heating was found to be around $0.03 \%$ for an applied DC current of 270 A. The shortterm intrinsic current dependence was found to be around $0.03 \%$ as well when comparing currents down to $30 \mathrm{~A}$ to the error determined at 270 A. Furthermore, small hysteresis was observed with respect to the direction of changing the current level, which is probably caused by different heating. The effect of AC distortion on the DC transresistance error was found to be negligible, or at least difficult to identify, due to the standard deviation of up to $0.10 \%$ at the highest frequencies. The intrinsic current dependence and the onset of the heating effect were also observed when exposing the sensor to two typical current patterns that were recorded during a trip between two successive underground train stations from Metro de Madrid.

The observed transresistance error of the high-current shunt investigated in this paper was around $0.10 \%$. The accuracy of the total energy measurement function of railway traction units, including current and voltage measurement and energy calculation, needs to be on the order of $0.2 \%$ to $1 \%$ [3]. Hence, the shunt under investigation would be just satisfactory for most metering applications on-board trains, since the errors (with uncertainties) of other components must be considered as well. However, it should be noted that the temperature in the laboratory was $(23.0 \pm 0.5){ }^{\circ} \mathrm{C}$ and the shunt was equipped with a cooling fan, whereas in practical railway applications the temperature can vary between, say, $-20^{\circ} \mathrm{C}$ and $+40{ }^{\circ} \mathrm{C}$, and active cooling of the shunt might be absent or less effective. In the future we will investigate the temperature dependence of the sensor under test using a temperature-controlled incubator.

The effect of AC distortion on the error of the shunt is shown to be negligible. It should be noted, however, that AC ripple might still influence the complete current measurement system. Modulation and spectral leakage might disturb the reading of the meter if it is not configured correctly. However, in this paper the focus is on current sensors only and the investigation of complete current measuring systems is left for future work.

Harsh conditions such as arching of the overhead lines or chopped waveforms when measuring the current in the rheostat when the train is braking can be implemented as a user defined waveform. However, such waveforms have not yet been tested so far.

From the results presented here, we conclude that the setup described in this paper is very useful to characterize DC current sensors for practical railway applications under realistic distortion conditions.

\section{ACKNOWLEDGEMENT}

The research leading to the results described in this paper is performed within the 16ENG04 MyRails project of the European Metrology Programme for Innovation and Research (EMPIR). The EMPIR initiative is co-funded by the European Union's Horizon 2020 research and innovation programme and the EMPIR participating states. We would like to thank Jorge Quintana Fernández for providing us with the current profiles measured in the underground traction unit of Metro de Madrid and for valuable discussions.

\section{REFERENCES}

[1] Arturo González-Gil, Roberto Palacin, P. Batty, and Jonathan Powell, "A systems approach to reduce urban rail energy consumption”, Energy Convers. Manage., vol. 80, pp. 509-524, April 2014.

[2] Commission Regulation (EU) No 1301/2014 of 18 November 2014 on the technical specifications for interoperability relating to the energy subsystem of the rail system in the Union.

[3] EN 50463-2:2012, "Railway applications - Energy measurement on board trains - Part 2: Energy measuring".

[4] H. E. van den Brom, G. Rietveld, and E. So, "Sampling Current Ratio Measurement System for Calibration of Current Sensors up to $10 \mathrm{kA}$ with $5 \cdot 10^{-6}$ Uncertainty", IEEE Trans. Instr. Meas, vol. 64, no. 6, pp. 1685-1691, June 2015.

[5] H. C. Appelo, M. Groenenboom, and J. Lisser, "The Zero-Flux DC Current Transformer - a High Precision Bipolar Wide-Band Measuring Device", IEEE Trans. Nucl. Sci., vol. 24, no. 3, pp. 1810-1811, June 1977.

[6] W. G. K. Ihlenfeld and M. Seckelmann, "Simple algorithm for sampling synchronization of ADCs," IEEE Trans. Instrum. Meas., vol. 58, no. 4, pp. 781-785, Apr. 2009.

[7] G. Rietveld, J. H. N. van der Beek, and E. Houtzager, "Accurate DC Current Ratio Measurements for Primary Currents up to 600 A", IEEE Trans. Instr. Meas, vol. 64, no. 11, pp. 3055-3061, Nov 2015.

[8] K. Lind, T. Sorsdal, and H. Slinde, "Design, Modeling, and Verification of High-Performance AC-DC Current Shunts from Inexpensive Components", IEEE Trans. Instr. Meas, vol. 57, no. 1, pp. 176-181, Jan. 2008.

[9] H. E. van den Brom, R. van Leeuwen, and R. Hornecker, "Characterization of a reference DC current sensor with AC distortion for railway applications", submitted to Conf. Prec. Elec. Meas., Paris, France, 8-13 July 2018. 